\title{
Intraocular Penetration of Orally and Intravenously Administered Fluoroquinolones in Non-Inflamed Eyes of the Patients Undergoing Vitrectomy Surgery
}

\author{
Alok Kumar Ravi ${ }^{\mathrm{a}}$, Nihar Ranjan Biswas*, ${ }^{\text {,b }}$, Thirumurthy Velpandian ${ }^{\mathrm{a}}$, Raju Sampangi ${ }^{\mathrm{c}}$, \\ Sat Pal Garg ${ }^{\mathrm{c}}$ and Supriyo Ghose ${ }^{\mathrm{d}}$
}

\begin{abstract}
${ }^{a}$ Department of Ocular Pharmacology and Pharmacy, ${ }^{b}$ Department of Pharmacology, ${ }^{c}$ Department of Medical Ophthalmology, Retina and Uvea, ${ }^{d}$ Department of Pediatric Ophthalmology and Oculoplasty, Dr. Rajendra Prasad Centre for Ophthalmic Sciences, All India Institute of Medical Sciences, New Delhi -110 029, India
\end{abstract}

\begin{abstract}
Purpose: This study evaluated intraocular penetration of fluoroquinolones (FQs) viz, ofloxacin, pefloxacin, lomefloxacin and sparfloxacin in aqueous, vitreous humors, and plasma after oral/intravenous administration in the subjects undergoing vitrectomy. Methods: Ofloxacin, pefloxacin, lomefloxacin and sparfloxacin were included for oral administration. Only ofloxacin and pefloxacin included for intravenous study. Each group was sub-divided into 3 time points (3, 6 and $12 \mathrm{hrs}$ post dose). For each time point, 8 eyes ( 8 patients) were included and a total of 144 patients were enrolled. Intra-operatively, $80-110 \mu \mathrm{l}$ aqueous, $100-250 \mu \mathrm{l}$ vitreous and $2 \mathrm{ml}$ of venous blood samples were collected. Plasma was separated and all samples were stored at $-70^{\circ} \mathrm{C}$ and subjected for quantification using HPLC and compared with their known $\mathrm{MIC}_{90}$ of common ocular pathogens. Results: In oral $400 \mathrm{mg}$ single dose, among all 4 FQs, ofloxacin showed a maximum concentration at 3 hours in aqueous $[0.98(0.12) \mu \mathrm{g} / \mathrm{ml}]$ and vitreous $[0.78(0.12) \mu \mathrm{g} / \mathrm{ml}]$. Intravenous infusion of $400 \mathrm{mg}$ ofloxacin also showed the similar levels in the humors of the eye. Despite low plasma levels, lomefloxacin showed higher aqueous to plasma (a/p) and vitreous to plasma (v/p) ratio. Conclusion: FQs 400mg (as iv and oral) as a single dose could not reach the desired therapeutic concentration (above $\mathrm{MIC}_{90}$ for all ocular pathogens) in aqueous and vitreous humors. The concentration reached in the humors may not be directly dependent on their plasma levels. Relatively non-polar FQs such as lomefloxacin and sparfloxacin showed better a/p and v/p ratios as compared to their polar analogs such as pefloxacin and ofloxacin.
\end{abstract}

Keywords: Intraocular penetretion, vitrectomy, fluoroquinolones, oral, intravenous.

\section{INTRODUCTION}

Bacterial endophthalmitis are rare but generally serious in nature. It occurs after intraocular surgery, penetrating ocular trauma, and corneal ulcer. Conventionally, antimicrobial drugs developed and approved for systemic infections are re-investigated for ocular infections [1]. The extent of antimicrobial drug absorption and resulting plasma concentration is governed by physiochemical and pharmacokinetic property of the drug. The intraocular availability of any drug is highly relevant to plasma concentration achieved after its absorption from the intestine and its distribution into the eye is based upon its physiochemical and transporter susceptibility in ocular barriers [1]. An effective antimicrobial prophylaxis before ocular surgeries would be of great benefit to the patients to reduce the chance of post surgical infection. Therefore, drugs reaching antimicrobial concentrations (above $\mathrm{MIC}_{90}$ of all ocular pathogens) into the ocular humors as a prophylactic measure as of now remains uncertain with available class of antimicrobial agents.

*Address correspondence to this author at the Department of Pharmacology, All India Institute of Medical Sciences, New Delhi -110 029, India; Tel: +91-011-26594897, 26190199; Fax: 26588663;

E-mail: nr_biswas@yahoo.com,nrbiswas@hotmail.com
The fluoroquinolones (FQs) have become widely used antibacterial agents in the treatment of ocular infections with topical, intravitreal and systemic routes of administration. In general, fluoroquinolones are reported to have good activity against Gram-negative and Gram-positive bacteria [2]. Ofloxacin, lomefloxacin, sparfloxacin and pefloxacin are used worldwide in many countries and were not effectively compared for their intraocular penetration in human as compared to their plasma concentration at various time intervals. As vitreous and aqueous drug penetration is mostly related to the plasma concentration, comparing the drug concentration in the humors with their respective plasma concentration at the same time was felt as a good indicator to evaluate their eye penetration properties across wellrecognized ocular barriers. Therefore, the purpose of our study was to comparatively evaluate the ocular penetration of ofloxacin, pefloxacin, sparfloxacin and lomefloxacin concentrations evidenced by aqueous and vitreous humor levels with respect to their plasma levels. The aqueous, vitreous humor and plasma samples were collected at various time intervals from patients undergoing vitrectomy surgical procedures after single oral administration of their respective commercial tablet formulations. This study was further extended to evaluate the impact of absorption affecting plasma concentration and subsequent ocular penetration of 
FQs, by directly administering ofloxacin and pefloxacin intravenously in similar patient group.

\section{MATERIALS AND METHODS}

\section{Materials}

Pure pefloxacin mesylate dihydrate, ofloxacin, lomefloxacin $\mathrm{HCl}$ and sparfloxacin were gifted by Smreeti organics, Solapur, India; Cipla Ltd., India; Plant organics Ltd., Hyderabad, India and Torrent pharmaceuticals, Ahmedabad, India respectively. HPLC grade acetonitrile (E. Merck [India] Limited, Mumbai), methanol (Ranbaxy fine chemicals Ltd., New Delhi, India), orthophosphoric acid (Spectrochem private Ltd., Mumbai, India) and potassium di-hydrogen orthophosphate (Central drug house (P) Ltd., Mumbai, India) were purchased. Fresh frozen plasma was supplied by blood bank of All India Institute of Medical Sciences, New Delhi.

\section{Drug Formulations}

Tablet ofloxacin 200mg (Oflin 200, Cadila Healthcare Ltd., Ahmedabad, India), pefloxacin 400mg (PEFLOX-400, Wockhardt Ltd., Nani Daman, India) were obtained from the commercial sources. Tablet lomefloxacin 400mg (Lomef, Torrent Pharmaceuticals, Ahdmedabad, India) and sparfloxacin 200mg (Sparquin 200, Aristo Pharmaceuticals, Mumbai, India) were procured from their respective source. Zanocin (ofloxacin I.V. $400 \mathrm{mg} / 100 \mathrm{ml}$ infusion) of Stancare Division of Ranbaxy Laboratories Ltd., Gurgaon, India and lfipef (pefloxacin I.V. $400 \mathrm{mg} / 100 \mathrm{ml}$ infusion) of J. B. Chemicals \& Pharmaceuticals Ltd., Mumbai, India were procured commercially.

\section{Methods}

This study was carried out with the approval of Standing Institutional Human Ethics Committee of All India Institute of Medical Sciences, New Delhi, India. This study was conducted in accordance with the declaration of Helsinki. Informed consent was obtained from each patient before participating in this study. Patients 18 years or older of both sex and undergoing vitrectomy for a variety of non-inflamed vitreo-retinal diseases were included. Patients with a known allergy to any of the fluoroquinolones, those who had received study fluoroquinolones either topical or oral form in the preceding two weeks and pregnant women were excluded from this study. Inflamed and infectious patients with any open periphery or posterior break were also excluded.

\section{Drug Schedule for Oral and Intravenous Administration}

A total number of one hundred and forty four patients were enrolled in this study with various ocular indications such as proliferative diabetic retinopathy with vitreous hemorrhage, proliferative vitreous retinopathy with total retinal detachment, idiopathic pre-retinal membrane and macular hole. Patients were divided in to six groups with 24 patients in each.

Ninety-six Subjects undergoing oral FQ administration were further divided into 4 drug groups. Each oral FQ drug group was further divided to have eight patients in each time point viz. 3, 6 and 12 hours respectively. Each drug group received $400 \mathrm{mg}$ dose of either ofloxacin (two tablets of
$200 \mathrm{mg}$ ) or pefloxacin or lomefloxacin or sparfloxacin with $150 \mathrm{ml}$ of water as single dose before surgery.

For subjects receiving drugs by intravenous route, the total of 48 enrolled patients were divided into two drugs group of 24 each. Pefloxacin $400 \mathrm{mg} / 100 \mathrm{ml}$ and ofloxacin $400 \mathrm{mg} / 100 \mathrm{ml}$ were administered as a slow intravenous infusion over the period of 30 minutes. Each drug group was further sub-divided into 3 time points having 8 patients each. Aqueous, vitreous humors and venous blood were collected at 3,6 and 12 hours following drug administration.

\section{Sample Collection}

Vitrectomy surgical procedure was done under local anesthesia by the ophthalmologists. From each patient, 50$110 \mu \mathrm{l}$ aqueous humor was aspirated through limbus area with the help of 30 gauge needle fitted with tuberculin syringe just before vitrectomy. For vitreous aspiration, a three-port pars plana procedure was adopted in patients posted for vitrectomy surgery. The infusion cannula was placed inferotemporally $4 \mathrm{~mm}$ from the limbus and secured with mattress Vicryl 6-0 suture. The endo-illuminator was inserted through the other upper port. Under direct visualization with the help of a fundus contact lens, 100$250 \mu 1$ of a vitreous humor sample was collected before turning on the infusion, from the mid vitreous cavity using a vitreous cutter attached to a $5 \mathrm{ml}$ syringe, which was used for manually aspirating the vitreous being cut by the cutter. Immediately, infusion cannula was connected to the infusion bottle, which subsequently maintains the intraocular pressure and prevents development of hypotony. Simultaneously vitrectomy cutter was removed from the vitreous cavity and rest of the vitreous from vitrectomy cutter tube was aspirated with distal end connected syringe. Vitreous was then transferred to sterile vial.

The $2 \mathrm{ml}$ blood sample was also withdrawn within 10 minutes of vitrectomy surgery and kept in anticoagulant tube. Samples were transported to the Department of Ocular Pharmacology and Pharmacy and plasma was separated by centrifugation at $1800 \mathrm{~g}$ for 10 minutes. All samples of aqueous, vitreous and plasma were stored immediately at $70^{\circ} \mathrm{C}$ deep freezer till analysis. Sampling times for the aqueous, vitreous and plasma were noted. Indication for surgery, types of procedure and preexisting medical conditions were also recorded.

\section{High Performance Liquid Chromatography Analysis of Fluoroquinolones}

\section{HPLC Instrumentation}

The HPLC equipment consisted of Surveyor PDA detector, autosampler and quaternary LC pump (Thermo Finnigan, USA). Luna Column C-18, $5 \mu, 250 \times 4.6 \mathrm{~mm}$

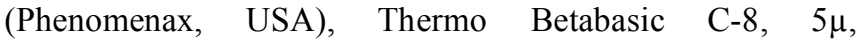
$100 \times 4.6 \mathrm{~mm}$ (Thermo Electron Corporation, USA) and Vydac C-18 monomeric, $5 \mu, 250 \times 4.6 \mathrm{~mm}$ (Grace, USA) were used for analytical separation and Chromquest ver. 4.1 software was used for HPLC data analysis.

The analytical separation of individual fluoroquinolones was achieved with different proportions of the mobile phase, which consisted of $0.02 \mathrm{M}$ potassium dihydrogen phosphate adjusted to $\mathrm{pH} 2.5$ and acetonitrile (Table 1). Solvents and 
Table 1. Conditions of HPLC Analysis of Different Fluoroquinolones

\begin{tabular}{|c|c|c|c|c|c|}
\hline $\mathbf{F} \mathbf{Q}_{\mathrm{s}}$ & $\begin{array}{c}\text { Mobile Phase } \\
(0.02 M \text { phosphate buffer :ACN) }\end{array}$ & Column & $\begin{array}{c}\lambda \max \\
(\mathbf{n m})\end{array}$ & $\begin{array}{c}\text { Flow } \\
\text { Rate } \\
\text { (ml/min) }\end{array}$ & $\begin{array}{c}\text { Total Run } \\
\text { Time } \\
\text { (mins) }\end{array}$ \\
\hline Ofloxacin & $76: 24$ & 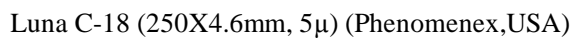 & 280 & 1 & 10 \\
\hline Pefloxacin & $87: 13$ & $\begin{array}{c}\text { Thermo Betabasic C-8 (100X4.6, 5 } \mu \text { ) } \\
\text { (Thermo Electron Corp.,USA) }\end{array}$ & 278 & 1 & 10 \\
\hline Sparfloxacin & $76: 24$ & $\begin{array}{l}\text { Vydac C-18 monomeric } \\
\qquad(250 \times 4.6 \mathrm{~mm}, 5 \mu)\end{array}$ & 298 & 1 & 10 \\
\hline Lomefloxacin & $78: 22$ & $\begin{array}{l}\text { Vydac C-18 monomeric } \\
\qquad(250 \mathrm{X} 4.6 \mathrm{~mm}, 5 \mu)\end{array}$ & 288 & 1 & 10 \\
\hline
\end{tabular}

buffer were filtered by $0.22 \mu$ filters (Millipore) and degassed with in-built on-line degasser and pumped at the flow rate of $1 \mathrm{ml} / \mathrm{min}$. The column temperature was kept at $25 \pm 1^{\circ} \mathrm{C}$. The ambient temperature was maintained in and out of the system. Twenty micro-liter of sample was injected from 96 well sample plates using auto-sampler and the analytical separation was achieved using RP-C18 and C-8 column of different make at particular wavelengths for individual fluoroquinolones as shown in the Table $\mathbf{1}$.

Stock solution $(1 \mathrm{mg} / \mathrm{ml})$ of ofloxacin, pefloxacin, sparfloxacin, and lomefloxacin was prepared separately in $10 \mathrm{ml}$ volumetric flask in pure methanol $(\mathrm{MeOH})$. Serial dilutions $(100-0.012 \mu \mathrm{g} / \mathrm{ml})$ were made in $50 \% \mathrm{MeOH}$ in water from stock solution. The area of the respective HPLC peaks was plotted against their concentration to get the linearity. Water spiking was used to plot the calibration curve for aqueous and vitreous sample. Ofloxacin, pefloxacin, sparfloxacin and lomefloxacin pure drug in human drug free plasma (obtained from Blood Bank of AIIMS) were used for plasma spiking and separate calibration curve was made.

\section{Sample Extraction Procedure}

Aqueous, vitreous and plasma samples were subjected for direct deproteinization method. Briefly, samples amounting to $100 \mu \mathrm{l}$ was mixed with $200 \mu \mathrm{l}$ of acetonitrile (ratio of 1:2) and subjected for vortexing and centrifugation at $7000 \mathrm{~g}$ for $15 \mathrm{~min}$. The supernatant (clear fluid) was carefully separated and kept in the HPLC autosampler 96 well plates for the estimation. Then, $20 \mu \mathrm{l}$ of the supernatant was injected into the HPLC column for the analytical separation and quantification.

\section{Statistical Analysis}

For the evaluation of significance within the same drug group, Student's paired t test was employed using the software Sigma Stat (Ver. 2) and p value $<0.05$ was considered for the level of significance. The results are expressed as Mean \pm SEM (Standard Error Mean).

\section{RESULTS}

Single dose of $400 \mathrm{mg}$ orally administered ofloxacin, pefloxacin, lomefloxacin and sparfloxacin or intravenously injected pefloxacin and ofloxacin showed detectable levels in all the samples (plasma, vitreous and aqueous) in the patients undergoing vitrectomy surgery in all studied time points. The mean age of patients with standard mean error and their resulting plasma, vitreous and aqueous concentration after oral or intravenous administration of studied fluoroquinolones are shown in the Table 2.

The vitreous and aqueous levels reached by all studied fluoroquinolones were found to be less than that of their respective plasma concentrations. Despite reaching higher plasma concentration in the pefloxacin oral as well as intravenous group the levels reached in the vitreous were low in all study points. While comparing the aqueous as well as vitreous penetration ratio of sparfloxacin and lomefloxacin, ofloxacin and pefloxacin showed poor blood to ocular penetration ratio. Interestingly, highest plasma concentration was not coincided with higher aqueous and vitreous concentration in all fluoroquinolones in the studied time points. In the group of lomefloxacin and sparfloaxcin higher vitreous and aqueous levels were found at the last time point studied (12 hours) after drug administration.

\section{DISCUSSION}

Fluoroquinolones are a class of synthetic antibacterial agents that were approved for ocular therapy in 1991. Ever since, they are used as a popular therapy for the treatment and prevention of various ocular infections [3]. Presently, fluoroquinolones are widely used as an antibacterial agent with topical, intravitreal and systemic routes of administration. These synthetic agents have broad-spectrum of activity, rapid bactericidal action and are cited to have good penetration into ocular tissues [4]. In general, fluoroquinolones such as norfloxacin, ciprofloxacin, ofloxacin, and lomefloxacin etc. have good activity against Gram-negative and Gram-positive bacteria. These agents are not reported to cause corneal or retinal toxicities when used within therapeutic range via topical or systemic administration.

Systemic administration of FQs can be a useful prophylactic measure after ocular trauma to prevent bacterial endophthalmitis if they are found to reach optimal levels in the non-inflamed eyes. In spite of reasonable penetration of systemic FQs into the humors of the eye, it is not guaranteed whether it is higher enough to reach the $\mathrm{MIC}_{90}$ of all ocular pathogens. Seemingly, increasing the dose of the drugs or 
Table 2. Concentrations of Each Drug Group Alongwith their Ratio in Aqueous and Vitreous Humour

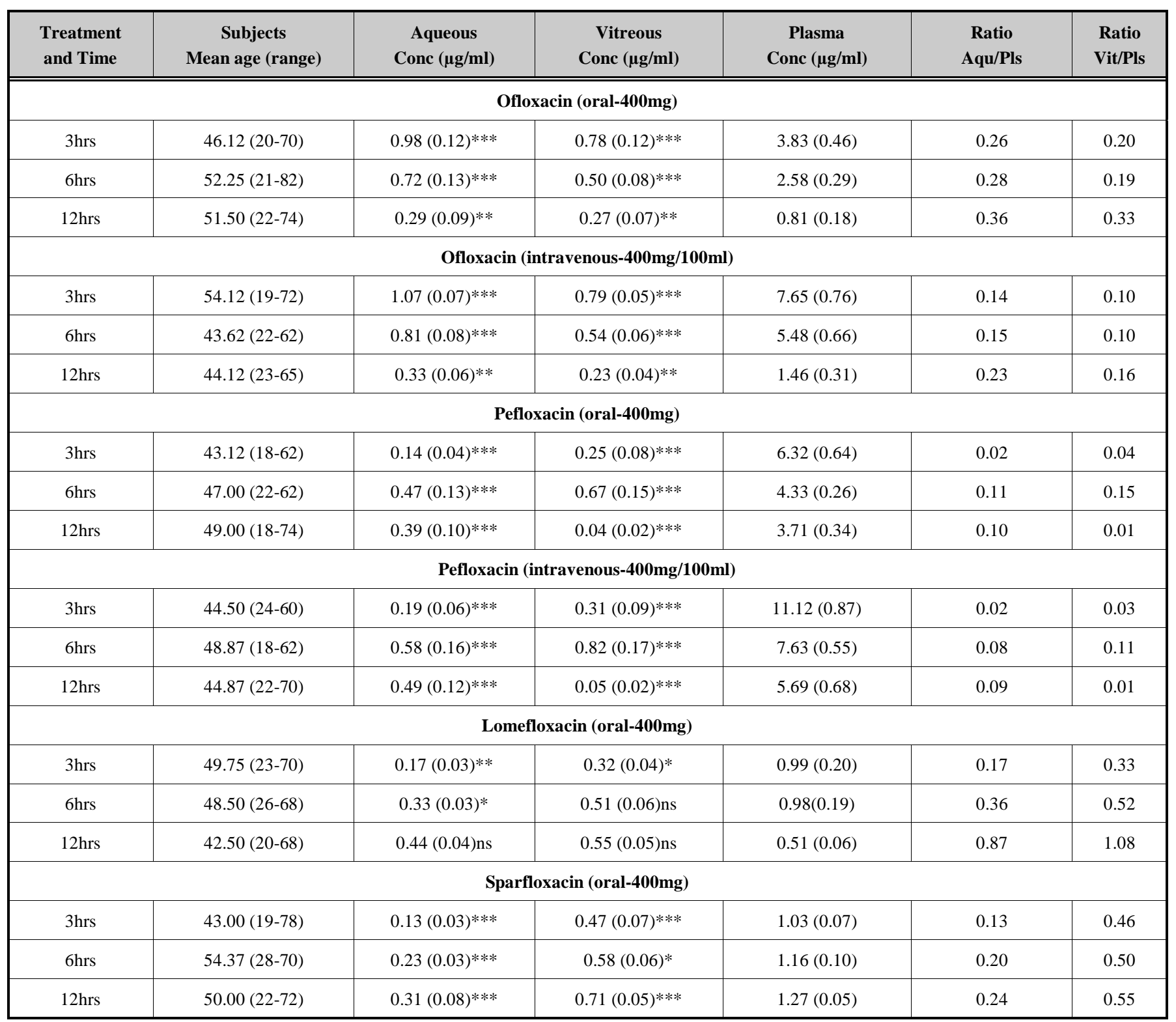

$*$ Represent the level of significance as compared to their respective plasma levels (paired Student ' $t$ ' ${ }^{*}=p<0.05, * *=p<0.01$ and $* * *=p<0.001$, ns $=$ not-significant $)$. Aqu/Pls $=$ Aqueous to plasma ratio, Vit/Pls = Vitreous to plasma ratio.

their frequency of dosage are the popular attempts in order to enhance the ocular penetration of antimicrobial agents for their expected usage as prophylaxis in non-inflamed eyes. Several studies have reported the individual ocular pharmacokinetics of systemically administered fluoroquinolones using the above strategies. However, due to the difference in the clinical set up and protocol used for these studies, it is often difficult to compare their ocular penetration with respect to their plasma concentration. Therefore, the necessity of present study comparing the ocular penetration of commonly used fluoroquinolones after their systemic (oral and intravenous) modes of administration was felt.

In the present study, after 400mg dosage, ofloxacin reached the highest concentration in the aqueous humor followed by pefloxacin, lomefloxacin and sparfloxacin respectively. Von Gunten et al. 1994 [5] studied the aqueous humor penetration of ofloxacin after oral administration at the dose of $200 \mathrm{mg}$ and reported that the 2 hours aqueous concentration $[0.38(0.15) \mu \mathrm{g} / \mathrm{ml}]$ was significantly lower than that of 12 hours aqueous concentration [0.58(0.24) $\mu \mathrm{g} / \mathrm{ml}]$. In contrast, the present study showed that 3 hours aqueous concentration $[0.98(0.12) \mu \mathrm{g} / \mathrm{ml}]$ was significantly $(\mathrm{p}<0.001)$ higher than 12 hours aqueous concentration $[0.29(0.09) \mu \mathrm{g} / \mathrm{ml}]$ after $400 \mathrm{mg}$ ofloxacin oral administration. Similarly, in the present study ofloxacin reached the highest concentration in the vitreous humor followed by pefloxacin, lomefloxacin and sparfloxacin respectively. Verbraeken et al. 1996 [6] studied the intraocular penetration of gentamicin and ofloxacin in human vitreous humor after systemic administration. They reported that ofloxacin levels were higher as compared to gentamicin but it did not reach the $\mathrm{MIC}_{90}$ levels of most organisms involved in bacterial endophthalmitis. The present study supports the findings of Verbraeken et al. 1996 [6]. 
Donnenfeld et al. 1997 [7] studied the intracorneal, aqueous humor and vitreous humor penetration of oral ofloxacin (400mg). Ten patients received an additional 3 doses of oral ofloxacin $400 \mathrm{mg}$, every 12 hours starting 36 hours before surgery. This study reported that the mean intracorneal, aqueous and vitreous humor levels of ofloxacin were 8.59, 2.77 and $2.55 \mu \mathrm{g} / \mathrm{ml}$ respectively. The higher ofloxacin levels reported in cornea, aqueous and vitreous humors could be due to the cumulative effect after repeated administration.

In the present study, after oral administration, pefloxacin reached the highest plasma concentration followed by ofloxacin, sparfloxacin and lomefloxacin respectively. Among the FQs evaluated in our study, only ofloxacin and pefloxacin were available for intravenous use. Therefore, they were included in this study to evaluate the intraocular penetration at 3,6 and 12 hours after the initiation of the slow infusion over the period of 30 minutes. Interestingly, higher plasma levels reached by pefloxacin after oral or intravenous administration were not accompanied by a proportionate increase in aqueous or vitreous levels. Similarly, increasing plasma concentration by direct intravenous infusion of ofloxacin did not significantly increase its corresponding aqueous or vitreous levels.

Although, intravenous pefloxacin reached $11.12 \mu \mathrm{g} / \mathrm{ml}$ as a plasma concentration at 3 hours post dose, the higher aqueous as well as vitreous humor levels were found at 6 hours only. Whereas, in ofloxacin group, 3 hours plasma concentrations showed increased aqueous as well as vitreous humor levels at 3 hours itself. This can only be explained by the transporter susceptibility of these agents at blood ocular barriers. Therefore, rather than increasing the dosage, increasing frequency may be having the beneficial cumulative effect similar to the findings that could be derived from the study of Donnenfeld et al. 1997 [7]. The lack of advantage on vitreous and aqueous levels by directly increasing plasma concentration via intravenous infusion, renders this option ineffective as a single dose mono-therapy for prophylaxis.

Oncel and Peyman 1993 [8] measured vitreous humor and serum levels of pefloxacin (400mg) after oral administration in twenty patients with non-inflamed eyes undergoing vitrectomy. Six hours after oral administration, an average level of $1.37 \mu \mathrm{g} / \mathrm{ml}$ of pefloxacin was measured in the vitreous humor samples. Where as, in our study 6 hours pefloxacin levels in the vitreous humor were found to be $0.7 \mu \mathrm{g} / \mathrm{ml}$. Salvanet-Bouccara et al. 1991 [9] studied the intraocular penetration of pefloxacin in 38 patients undergoing extracapsular cataract extraction. Patients were treated for three days with $400 \mathrm{mg}$ of pefloxacin every 12 hours (the first dose being $800 \mathrm{mg}$ ). Their results showed that the mean maximum concentration of pefloxacin reached $7.69(3.50) \mu \mathrm{g} / \mathrm{ml}$ at 6 hours after the last dose in the aqueous humor and 4.59(3.15) $\mu \mathrm{g} / \mathrm{g}$ in the lens at 12 hours after the last dose. In our study, 12 hours after the oral administration of pefloxacin $400 \mathrm{mg}$ achieved the aqueous humor levels of $0.39 \mu \mathrm{g} / \mathrm{ml}$. This shows that the 9 fold higher aqueous humor levels achieved by Salvanet-Bouccara et al. 1991 [9] could be due to initial higher dose followed by multiple dosing for two days subsequently. This study further supports the effect of cumulative dosing on the drug levels in humors of the eye.
Cochereau-Massin et al. 1993 [10] evaluated the efficacy and ocular penetration of sparfloxacin in experimental Streptococcal endophthalmitis in rabbits. After systemic administration in rabbits, the maximum concentration of sparfloxacin in serum was $5.6 \mu \mathrm{g} / \mathrm{ml}$ and the half-life was 7.5 hours. Sparfloxacin reported to exhibit very good penetration ratios in the vitreous humor (54\%), cornea (76\%), and lens $(36 \%)$. In the vitreous humor, the levels of sparfloxacin remained greater than the MICs for most Gram-positive cocci for up to 18 hours. In our human study, single dose sparfloaxcin $(400 \mathrm{mg}$ ) reached vitreous humor levels of $0.71(0.05) \mu \mathrm{g} / \mathrm{ml}$ at 12 hours after administration. Among the fluoroquinolones studied, sparfloxacin showed highest level of $0.71 \mu \mathrm{g} / \mathrm{ml}$ at 12 hours after administration, which is above the $\mathrm{MIC}_{90}$ of most common pathogens causing endophthalmitis in humans viz Streptococcus pyrogens, Streptococcus pneumoniae, Staphylococcus epidermidis and aureus [10]. However, this concentration may not be adequate to inhibit organisms like Pseudomonas aeuroginosa, Bacillus gragilis, Chlamydia difficile and atypical organisms like Chlamydia pneumonia etc. Therefore, considering sparfloxacin as a mono therapy for the prophylaxis in ocular trauma is not recommended. However, considering the slow raise in vitreous and aqueous levels observed along with time in lomefloxacin and sparfloxacin groups in the present study, further studies with multiple dosing to evaluate their corresponding increase in aqueous and vitreous levels are required. In the present study, the drug levels were studied in non-inflamed eyes, where as, the effect of inflammation induced breakage of ocular barriers [11,12] needs further studies.

To conclude, this study was conducted to evaluate the ocular penetration of orally and intravenously given fluoroquinolones in the patients undergoing ocular surgeries. Among the single dose of FQs studied, ofloxacin showed the highest concentration in the aqueous and vitreous humors after the $400 \mathrm{mg}$ oral/intravenous administration. Despite higher plasma levels of pefloxacin after oral and intravenous administration, the aqueous and vitreous concentrations were low as compared to ofloxacin. However, the over all aqueous and vitreous levels reached by ofloxacin over the period of 12 hours (after a monothearpy) in the non-inflamed eyes were not found to provide the required antimicrobial cover against all common ocular pathogens. By increasing plasma concentration (via intravenous infusion) neither pefloxacin nor ofloxacin resulted in proportionate rise in their aqueous or vitreous levels. This study further reveals that ocular barrier susceptibility of fluoroquinolones may be having some degree of relevance to their lipophilicity as comparatively more non-polar drugs like sparfloxacin and lomefloxacin gained higher vitreous to plasma and aqueous to plasma ratios along with time as compared to more polar drugs like pefloxacin and ofloxacin. Increasing frequency would be having benefit rather than increasing plasma concentrations in the studied fluoroquinolones. Therefore, as a prophylactic monotherapy of any of the above fluoroquinolones as a single dose requires caution. Further studies are required to know the impact of multidose therapy on the intraocular penetration of the above studied fluoroquinolones in non-inflamed eyes for the prophylactic use in the ocular emergencies. 


\section{ACKNOWLEDGEMENT}

This study was supported by the Department of Science and Technology (DST) through, the grant SP/SO/B-08/2000, Ministry of Science and Technology, Govt. of India. This study was presented at Indian Eye Research Group meeting (IERG) XVI held at Hyderabad on $28^{\text {th }}$ July 2007.

\section{REFERENCES}

[1] Velpandian, T. Intraocular penetration of antimicrobial agents in ophthalmic infections and drug delivery strategies. Expert. Opin. Drug Deliv., 2009, 6, 255-270.

[2] Smith, A.; Pennefather, PM.; Kaye, SB.; Hart, CA. Fluoroquinolones: Place in ocular therapy. Drugs, 2001, 61, 747761.

[3] Andersson, MI.; MacGowan AP. Development of the quinolones. J. Antimicrob. Chemother., 2003, 51, 1-11.

[4] Stroman, DW.; Dajes, JJ.; Cupp, GA.; Schlech BA. In vitro and in vivo potency of moxifloxacin and moxifloxacin ophthalmic solution $0.5 \%$, a new topical fluoroquinolone. Surv. Ophthalmol., 2005, 50, S16-31.

[5] Von Gunten, S.; Lew, D.; Paccolat, F.; Vaudaux, P.; Brazitikos, PD. Aqueous humor penetration of ofloxacin given by various routes. Leuenberger PM. Am. J. Ophthalmol., 1994, 117, 87-89.
[6] Verbraeken, H.; Verstraete, A.; Van de Velde, E.; Verschraegen, G. Penetration of gentamicin and ofloxacin in human vitreous humour after systemic administration. Graefes Arch. Clin. Exp. Ophthalmol., 1996, 234, S59-65.

[7] Donnenfeld, ED.; Perry, HD.; Snyder, RW.; Moadel, R.; Elsky, M.; Jones, H. Intracorneal, aqueous humour, and vitreous humour penetration of topical and oral ofloxacin. Arch. Ophthalmol., 1997, 115, 173-176.

[8] Oncel, M.; Peyman, GA. Intravitreal penetration of oral pefloxacin in humans. Intl. Ophthalmol., 1993, 17, 217-222.

[9] Salvanet-Bouccara, A.; Montay, G.; Fisch, A.; Forestier, F.; Meziane, D.; Lafaix, C.; Prieur, MB. Diffusion of pefloxacin in the aqueous humour and crystalline lens after repeated oral administration in man. J. Fr. Ophtalmol., 1991, 14, 260-264.

Cochereau-Massin， I.; Bauchet，J.; Marrakchi-Benjaafar，S.; Saleh-Mghir, A.; Faurisson F.; Vallois JM.; Vallee E.; Pocidalo JJ. Efficacy and ocular penetration of sparfloxacin in experimental streptococcal endophthalmitis. Antimicrob. Agents Chemother. 1993, 37, 633-636.

Alfaro, DV.; Hudson, SJ.; Rafanan, MM.; Moss, ST.; Levy, SD. The effect of trauma on the ocular penetration of intravenous ciprofloxacin. Am. J. Ophthalmol., 1996, 122, 678-683.

[12] Behrens-Baumann, W. Absorption of topically administered ciprofloxacin, ofloxacin and gentamicin in the inflamed rabbit eye. Ophthalmologica, 1996, 210, 119-122.

(C) Ravi et al.; Licensee Bentham Open.

This is an open access article licensed under the terms of the Creative Commons Attribution Non-Commercial License (http://creativecommons.org/licenses/by-nc/3.0/) which permits unrestricted, non-commercial use, distribution and reproduction in any medium, provided the work is properly cited. 